Research Paper

\title{
Neoadjuvant chemoradiotherapy or radiotherapy in patients aged 75 years or older with locally advanced rectal cancer
}

\author{
Xiaoliang Liu ${ }^{1}$, Junjie Wang ${ }^{2}, \mathrm{Ke} \mathrm{Hu}^{1 * \bowtie}$, Fuquan Zhang ${ }^{1 * \bowtie}$, Xiaorong Hou ${ }^{1}$, Yi Xiao $^{3}$, Xin Lian ${ }^{1}$, Shuai Sun ${ }^{1}$, \\ Zhikai Liu'1, Junfang Yan ${ }^{1}$, Zheng Miao ${ }^{1}$ \\ 1. Department of Radiation Oncology, Peking Union Medical College Hospital, Chinese Academy of Medical Sciences \& Peking Union Medical College, \\ Beijing, the People's Republic of China. \\ 2. Department of Gynecological Oncology, Qingdao Center Hospital, Qingdao, Shandong, the People's Republic of China. \\ 3. Department of Surgery, Peking Union Medical College Hospital, Chinese Academy of Medical Sciences \& Peking Union Medical College, Beijing, the \\ People's Republic of China. \\ *Ke Hu and Fuquan Zhang contributed equally to this work.
}

$\square$ Corresponding authors: Ke Hu, Department of Radiation Oncology, Peking Union Medical College Hospital, Chinese Academy of Medical Sciences \& Peking Union Medical College, NO.1 Shuaifuyuan Wangfujing, Dongcheng District, Beijing, China, 100730. Tel: 86-10-6915-5487; Fax: 86-10-6512-4875; Email: huk@pumch.cn Or Fuquan Zhang, Department of Radiation Oncology, Peking Union Medical College Hospital, Chinese Academy of Medical Sciences \& Peking Union Medical College, NO.1 Shuaifuyuan Wangfujing, Dongcheng District, Beijing, China, 100730. Tel: 86-10-6915-5485; Fax: 86-10-6512-4875; Email: Zhangfq@pumch.cn

(C) The author(s). This is an open access article distributed under the terms of the Creative Commons Attribution License (https://creativecommons.org/licenses/by/4.0/). See http://ivyspring.com/terms for full terms and conditions.

Received: 2019.11.10; Accepted: 2020.02.26; Published: 2020.03.15

\begin{abstract}
Background: To evaluate the efficacy and treatment related morbidity of neoadjuvant chemoradiotherapy or radiotherapy in elderly patients (aged 75 years or older) with locally advanced rectal cancer (LARC).

Methods: We reviewed clinical records of elderly patients with LARC treated with neoadjuvant chemoradiotherapy or radiotherapy between January 2008 and June 2017 at our institute. A dose of 45-50Gy in 25 fractions was delivered to pelvis. The primary tumor received a dose of 55Gy concomitantly for patients receiving intensity modulated radiotherapy (IMRT). The concurrent chemotherapy included capecitabine alone and capecitabine plus oxaliplatin (Xelox). Surgery was performed for suitable patients at least 6 weeks after neoadjuvant treatment. Overall survival (OS), disease specific survival (DSS), disease free survival (DFS) and local control (LC) were calculated with Kaplan-Meier method.

Results: A total of 85 patients were enrolled in this study, the median age was 80 years old (range: 75-90 years). After neoadjuvant treatment, surgery was performed in 56 patients $(65.9 \%)$. Downstaging rate was $85.7 \%(48 / 56)$ with $\mathrm{T}$ downstaging in 35 patients $(62.5 \%)$ and $\mathrm{N}$ downstaging in 36 patients $(64.3 \%)$. Twelve patients $(21.4 \%)$ obtained pathological complete response (PCR). The incidence of grade 3 or greater acute hematological, gastrointestinal and genitourinary toxicities were $10.7 \%, 5.2 \%$ and $1.8 \%$, respectively. Seven patients (12.5\%) experienced postoperative complications. The median follow-up duration was 35.7 months (range: $4.3-100.3$ months), The 3-year and 5-year OS, DSS, DFS, LC were $68.9 \%$ and $47.2 \%, 75.8 \%$ and $60.4 \%$, $68.2 \%$ and $56.1 \%, 83.9 \%$ and $78.3 \%$, respectively.

Conclusion: In patients aged 75 years or older with LARC, neoadjuvant chemoradiotherapy followed by surgery was well tolerated with promising survival outcomes, which should be strongly suggested if medically suitable.
\end{abstract}

Key words: rectal cancer, elderly, neoadjuvant, radiotherapy, chemotherapy

\section{Background}

Surgery, radiotherapy and chemotherapy are the primary treatment methods for patients with locally advanced rectal cancer (LARC). Compared with other modalities, neoadjuvant chemoradiotherapy followed by total mesorectal excision (TME) achieved better survival outcomes and fewer treatment related 
toxicities [1-5]. Therefore, this method has been identified as the standard treatment for patients with LARC.

As life expectancy increases, more and more elderly people suffer from rectal cancer [6]. With the increasing age, patients are more prone to suffer comorbidities and treatment related morbidities [7-10]. In a retrospective study [11], patients older than 75 years had worsen comorbidity status and more medical complications than those younger than 75 years. A report from ACCOR 12/PRODIGE 2 phase III trial revealed that elderly patients $(\geq 70$ years) with LARC experienced more G3/4 toxicities than younger patients ( $<70$ years) when performing preoperative chemoradiotherapy $(25.6 \%$ vs $15.8 \%$, $\mathrm{P}=0.01$ ) [12]. Another study compared surgery related morbidities between older and younger patients [13], the results showed greater intraoperative blood loss and a higher surgical conversion rate in older patients $(\geq 70$ years). All these negative effects may compromise the efficacy of treatment.

To achieve promising results, many clinical trials usually exclude elderly patients from the study group. In the study of FFCD9203 [2], all eligible patients were limited to younger than 75 years and with WHO performance status of 0 or 1 . In a phase III randomized trial comparing two neoadjuvant therapies for LARC [14], patients older than 75 years were excluded from the study. The CAO/ARO/ AIO-94 randomized phase III trial also only involved patients younger than 75 years [3]. These milestone studies all underrepresented elderly patients with LARC. The treatment options for elderly patients with LARC are mainly extrapolated from results concluded with relative younger patients. The best treatment choice for this group of patients is still unclear.

This retrospective study was designed to evaluate the tolerance and efficacy of neoadjuvant chemoradiotherapy or radiotherapy in elderly patients with LARC.

\section{Methods}

\section{Patients selection}

After receiving approval from the Institutional Review Board (IRB) of Peking Union Medical College Hospital, we reviewed the clinical records of patients with LARC treated with neoadjuvant chemoradiotherapy or radiotherapy from January 2008 to June 2017 at our institute. The inclusion criteria were as follows: histologically proven rectal cancer; with age $\geq 75$ years; with locally advanced stage (cT3+ or $\mathrm{cN}+$ ); scheduled to receive neoadjuvant chemoradiotherapy or radiotherapy. The pretreatment evaluation included complete history and physical examinations, digital rectal examination (DRE), complete blood counts and biochemical analysis, tumor markers (CEA and CA199), colonoscopy, thoracic and abdominal computed tomography (CT), rectal magnetic resonance imaging (MRI) and transrectal ultrasound. The positron emission tomography/CT (PET/CT) were not routinely used.

\section{Radiotherapy}

As described in our previous study [15], all eligible patients received pelvic irradiation with 3 dimensional conformal radiotherapy (3D-CRT) or intensity modulated radiotherapy (IMRT). Clinical target volume (CTV) included the complete mesorectum and pelvic lymph node region (presacral, internal iliac and obturator lymph node). Gross tumor volume (GTV) consisted of the primary tumor (GTV-T) and the positive pelvic lymph nodes (GTV-N). Positive lymph node was defined as short diameter $\geq 1 \mathrm{~cm}$ on MRI or confirmed by PET/CT. Planning clinical target volume (PCTV) was defined as CTV plus a $6-10 \mathrm{~mm}$ margin. A margin of $5 \mathrm{~mm}$ was added to GTV to form the planning gross tumor volume (PGTV).

A dose of 45-50Gy in 25 fractions was delivered to at least $95 \%$ of the PCTV. For patients receiving IMRT, at least $95 \%$ of the PGTV was escalated to 55Gy in 25 fractions with a simultaneously integrated boost (SIB) technique.

All patients treated with IMRT received image guidance at our institute. Patients who received volumetric modulated arc radiotherapy (VMAT) underwent cone beam computed tomography (CBCT) weekly. Daily on-board megavoltage CT (MVCT) was performed for patients receiving helical tomotherapy (HT).

\section{Chemotherapy}

Concurrent chemotherapy was scheduled to perform for eligible patients. Regimens included oral capecitabine $\left(825 \mathrm{mg} / \mathrm{m}^{2}, \mathrm{~d} 1-14\right)$ with or without oxaliplatin $\left(130 \mathrm{mg} / \mathrm{m}^{2}, \mathrm{~d} 1\right.$, Xelox). All patients were scheduled for two cycles of concurrent chemotherapy. Adjuvant chemotherapy was based on the postoperative pathological examinations and patients' conditions.

\section{Surgery}

Before surgery, rectal MRI and/or transrectal ultrasound were performed for patients to reevaluate the stage and possibility of resection. Whether to perform the surgery and the surgery modalities depended on patients' conditions, preferences and the attending surgeons. Surgery was generally conducted six to eight weeks after the neoadjuvant treatment. Patients with low rectal cancer who received 
sphincter-preservation TME receiving prophylactic ileostomy. Transanal endoscopic microsurgery (TEM) was an alternative for some patients with clinical complete response (cCR), very low rectal cancer $(<3 \mathrm{~cm}$ from the anal verge) and a strong desire for sphincter-preserving. "Watch-and-wait" approach was also a choice for patients with cCR.

\section{Follow-up and toxicity evaluation}

After surgery, patients received regular follow-up every three months in the first two years, then every six months during the next 3-5 years and once a year thereafter. The routine follow-up examinations included DRE, complete blood counts, liver and renal functions, carcinoembryonic antigen (CEA) and carbohydrate antigen 19-9 (CA19-9), colonoscopy, thoracic and abdominal CT, pelvic MRI. PET/CT was not recommended unless suspect of disease relapse. Acute neoadjuvant chemoradiotherapy related toxicities were assessed with Common Terminology Criteria for Adverse Events, version 3.0 (CTCAE 3.0).

\section{Statistical analyses}

Overall survival (OS) was defined as time from the start of the treatment to the date of death or last follow-up. Disease specific survival (DSS) referred to the date from the start of treatment to the death date due to the disease or the time of the last follow-up. Disease free survival (DFS) was counted from the start of the treatment to the time of any disease relapse or the last follow-up. The time from the start of treatment to the date of local recurrence or last follow-up was defined as local control (LC). OS, DSS, DFS and LC were calculated with Kaplan-Meier methods. All statistical analyses were performed with SPSS 23.0 software (SPSS Inc, Chicago, IL, USA). A two side $\mathrm{P}$ value of $<0.05$ was defined as statistically significant.

\section{Results}

\section{Patients' characteristics}

From January 2008 to June 2017, eighty-five elderly patients with LARC were scheduled for neoadjuvant chemoradiotherapy or radiotherapy at our institute. The median age was 80 years old (range: 75-85 years). All patients were diagnosed with rectal adenocarcinoma, with 42 patients $(49.4 \%)$ in the low rectum and 43 patients $(50.6 \%)$ in the middle rectum. Seventy-nine patients $(92.9 \%)$ had clinical T3 or T4 stage. Positive lymph nodes were involved in 61 patients $(71.8 \%)$. The detailed information of patients' characteristics is listed in Table 1.
Table 1. Characteristics of elderly patients with locally advanced rectal cancer.

\begin{tabular}{ll}
\hline Characteristics & No. (\%) \\
\hline Total & 85 \\
Age & Median: 80 (range: $75-90$ years) \\
Gender & \\
Male & $61(71.8 \%)$ \\
Female & $24(28.2 \%)$ \\
Tumor differentiation (pretreatment) & \\
Poorly & $9(10.6 \%)$ \\
Moderately & $43(50.6 \%)$ \\
Well & $9(10.6 \%)$ \\
Undefined & $24(28.2 \%)$ \\
Tumor location & \\
Low (<5cm) & $42(49.4 \%)$ \\
Middle (5-10cm) & $43(50.6 \%)$ \\
T stage (pretreatment) & \\
T1 & $2(2.4 \%)$ \\
T2 & $4(4.7 \%)$ \\
T3 & $73(85.8 \%)$ \\
T4 & $6(7.1 \%)$ \\
N stage (pretreatment) & \\
N0 & $24(28.2 \%)$ \\
N1 & $39(45.9 \%)$ \\
N2 & $22(25.9 \%)$ \\
Concurrent chemotherapy & \\
None & $12(14.1 \%)$ \\
Capecitabine & $54(63.5 \%)$ \\
Xelox & $19(22.4 \%)$ \\
Radiotherapy & \\
3D-CRT & $7(8.2 \%)$ \\
VMAT & $65(76.5 \%)$ \\
HT & $13(15.3 \%)$ \\
Surgery & $29(34.1 \%)$ \\
None & $48(56.5 \%)$ \\
TME & $35(72.9 \%)$ \\
$\quad$ Dixon & $10(20.8 \%)$ \\
$\quad$ Miles & $2(4.2 \%)$ \\
$\quad$ Partmann & $1(2.1 \%)$ \\
TEM & $8(9.4 \%)$ \\
\hline Abbreviations 3D-CRT: 3-dimensional conformal radiotherapy, VMAT: volumetric \\
modulated arc radiotherapy, HT: helical tomotherapy, TME: total mesorecal \\
excision, TEM: transanal endoscopic microsurgery, Xelox: capecitabine plus \\
oxaliplatin. & \\
&
\end{tabular}

\section{Neoadjuvant treatment}

All patients completed the radiotherapy procedure with 3D-CRT in seven patients $(8.2 \%)$, VMAT in 65 patients $(76.5 \%)$ and HT in 13 patients $(15.3 \%)$. The median radiotherapy duration was 35 days (range: $30-50$ days). Four patients (5.9\%) had prolonged radiotherapy because of grade 3 or 4 hematological toxicity. One patient suffered stenocardia when he finished 11 fractions of radiotherapy, and he received symptomatic treatment and complete cardiac examination in another specialized hospital. This patient completed the rest fractions after recovery. However, detailed treatment information about his heart disease was not available due to the retrospective nature and information sharing reason.

Twelve patients $(14.1 \%)$ didn't receive concurrent chemotherapy due to poor healthy conditions or patients' refusal. Fifty-four (63.5\%) and nineteen patients $(22.4 \%)$ underwent capecitabine alone and Xelox, respectively. 


\section{Surgery}

After neoadjuvant treatment, sixty-two patients received reevaluation of the tumor stage. Clinical complete response (cCR) occurred in 11 patients $(17.7 \%)$. Among them, seven patients received a "watch and wait" approach instead of definitive surgery, two patients had TME surgery, whereas the other two patients underwent TEM surgery.

A total of 56 patients $(65.9 \%)$ received surgery after neoadjuvant treatment. Forty-eight patients underwent TME surgery, including Dixon in 35 patients $(72.9 \%)$, Miles in 10 patients $(20.8 \%)$. Hartmann in two patients $(4.2 \%)$, and Parks in one patient $(2.1 \%)$. While TEM surgery was performed in eight patients. For patients who had TME, laparoscopic surgery was performed in 41 patients $(85.4 \%)$, and open surgery was only in seven patients $(14.6 \%)$. Sphincter-preserving surgery was performed in 44 patients $(78.6 \%)$.

Twenty-nine patients (34.1\%) didn't undergo surgery for several reasons. Seven patients with CCR received a "watch-and-wait" strategy, two patients who were not suitable for surgery had a primary tumor boost irradiation of $10 \mathrm{~Gy}$ in 5 fractions, the other 20 patients refused surgery due to personal reasons.

\section{Treatment related toxicities and complications}

Acute toxicity was defined as toxicities occurred during treatment and within three months after treatment. A total of 56 patients were available for scoring neoadjuvant treatment related toxicities, in which nine patients (16.8\%) suffered grade 3 or greater toxicities. The incidence of acute grade 3 or greater acute hematologic, gastrointestinal and genitourinary toxicity were $10.7 \%, 5.2 \%$ and $1.8 \%$, respectively. Seven patients $(12.5 \%)$ experienced postoperative complications including anastomotic fistula, ileus and abdominal infection (Table 2). There was no treatment related death in our study.

\section{Pathological evaluation}

After neoadjuvant treatment and surgery, pathological examination identified forty-eight patients (48/56, 85.7\%) with pathological downstaging including $\mathrm{T}$ downstaging in 35 patients $(62.5 \%)$ and $\mathrm{N}$ downstaging in 36 patients (64.3\%). Pathological complete response (pCR) occurred in 12 patients, which accounted for $21.4 \%$ of patients receiving surgery. Of the 39 patients with $\mathrm{N}+$ stage before treatment, negative lymph node was observed in 33 patients $(84.6 \%)$.

\section{Survivals and patterns of failure}

The median follow-up duration was 35.7 months (range: 4.3-100.3 months), twenty-three patients died of rectal cancer, whereas eight patients died due to other comorbidities during the follow-up. The 3-year OS, DSS, DFS and LC were $68.9 \%, 75.8 \%, 68.2 \%$ and $83.9 \%$, respectively, the estimated 5-year OS, DSS, DFS and LC were $47.2 \%, 60.4 \%, 56.1 \%$ and $78.3 \%$, respectively (Figure 1-4).

By the end of last follow-up, twenty-eight patients (32.9\%) suffered disease recurrence including ten patients $(11.8 \%)$ with local relapse only, fifteen patients $(17.6 \%)$ with distant metastasis only and three patients $(3.5 \%)$ with both local relapse and distant metastasis. Lung and liver were the most prevalent organs for distant metastasis.

Table 2. Treatment related toxicities and complications

\begin{tabular}{llll}
$\begin{array}{l}\text { nCRT toxicity } \\
\text { (56 patients available) }\end{array}$ & $\begin{array}{l}\text { Grade 3-4 } \\
\text { No. (\%) }\end{array}$ & $\begin{array}{l}\text { Surgery complications } \\
\text { (56 patients) }\end{array}$ & No. (\%) \\
\hline Hematologic & $6(10.7 \%)$ & Anastomotic fistula & $2(3.6 \%)$ \\
Gastrointestinal & $3(5.2 \%)$ & Ileus & $3(5.4 \%)$ \\
Genitourinary & $1(1.8 \%)$ & Infection & $3(5.4 \%)$ \\
\hline
\end{tabular}

Abbreviations nCRT: neoadjuvant chemoradiotherapy.

Notes: Some patients suffered more than one kind of toxicity or complication.

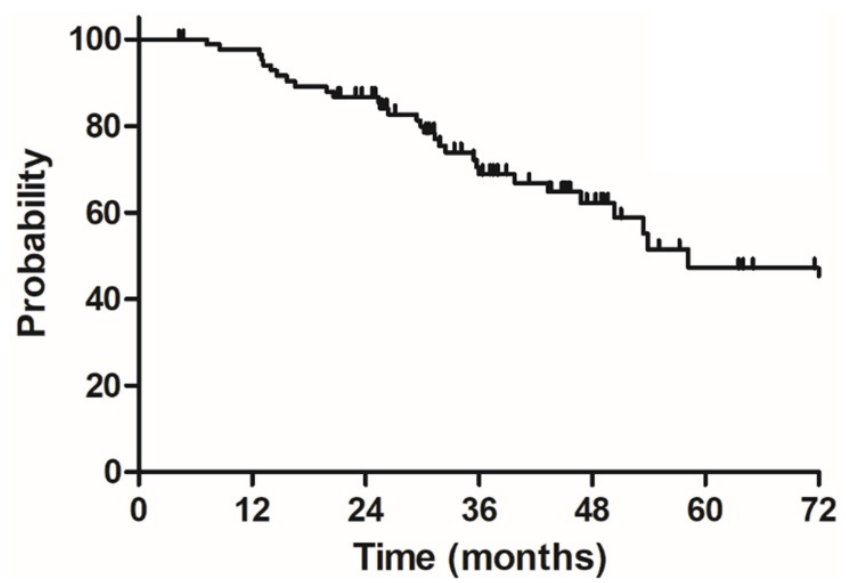

Figure 1. Overall survival (OS) for 85 elderly patients with LARC.

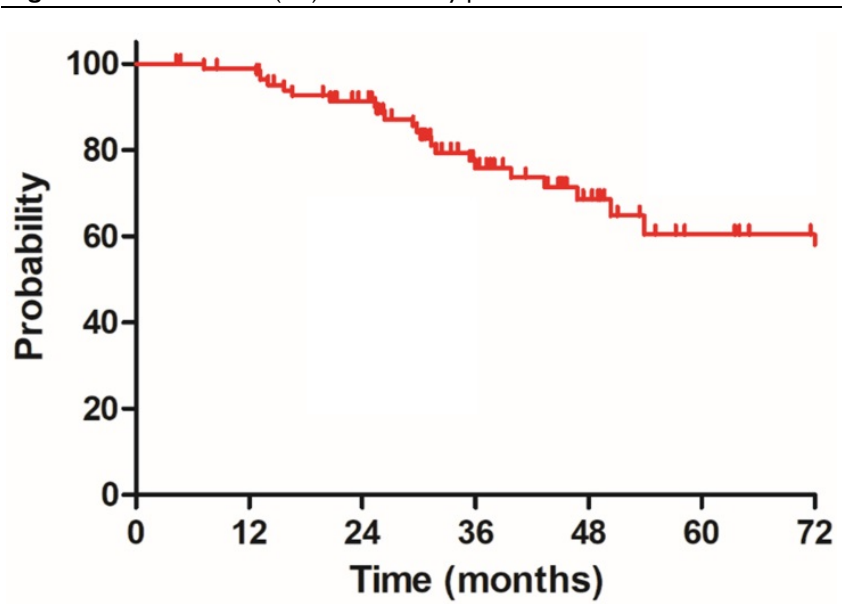

Figure 2. Disease specific survival (DSS) for 85 patients with LARC. 


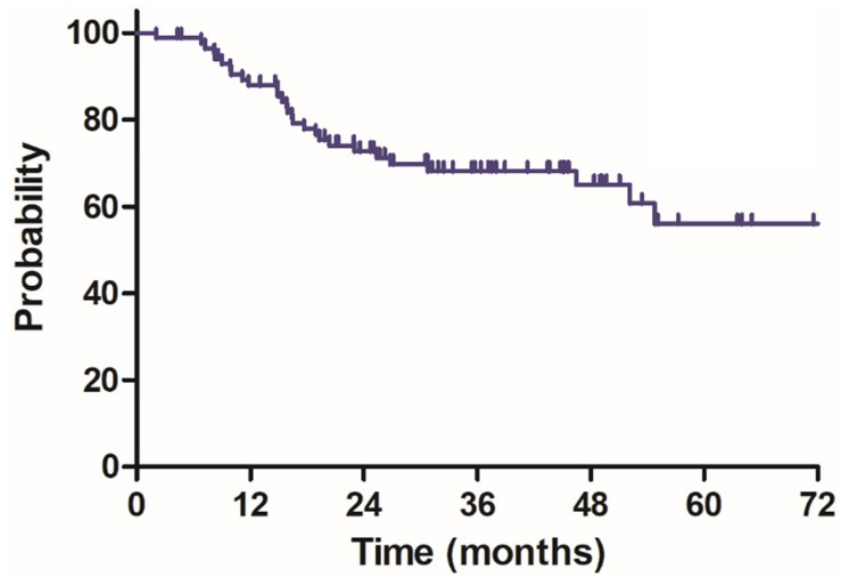

Figure 3. Disease free survival (DFS) for 85 patients with LARC.

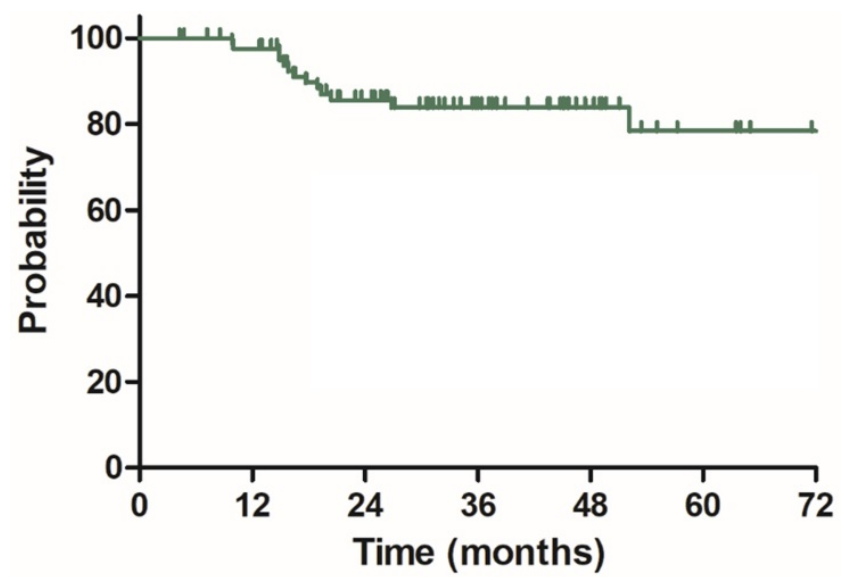

Figure 4. Local control (LC) for 85 patients with LARC.

\section{Discussion}

The best treatment option for elderly patients with LARC remained controversial due to lacking in prospective clinical data $[16,17]$. Though neoadjuvant chemoradiotherapy combined with TME surgery is the current standard treatment, many elderly patients can't finish the whole treatment procedure because of comorbidities, treatment related morbidities and other personal reasons $[7,9,16,18-20]$. Our study enrolled 85 patients at least 75 years with LARC. Fifty-six patients $(65.9 \%)$ completed the scheduled neoadjuvant treatment and surgery procedure. We achieved promising survival outcomes with acceptable treatment related toxicities and complications.

Many studies have reported the incidence of neoadjuvant chemoradiotherapy related toxicity in elderly patients with LARC [12, 19-22]. Tougeron D, et al [20] retrospectively evaluated safety of chemoradiotherapy in 125 patients with LARC over 70 years, about $15 \%$ of enrolled patients developed G3+ adverse events. In the study of ACCOR 12/PRODICE 2 phase III trial [12], the incidence of severe grade $3 / 4$ preoperative chemoradiotherapy related toxicity in elderly patients ( $\geq 70$ years) was $25.6 \%$, while it was only $15.8 \%$ in younger patients ( $<70$ years). A case-matched control study from Korea also showed higher rate of G3+ acute hematologic toxicity in elderly patients ( $\geq 70$ years), compared with that in patients younger than 70 years $(16.1 \%$ vs $9.0 \%)$ [21]. In our study, nine patients $(16.1 \%)$ developed G3/4 neoadjuvant treatment related toxicities, which was comparable with the above studies. Compared with our previous report [15] which included patients with a median age of 59 years (range: 50-67 years), patients in the present study also experienced more G3+ toxicities. Based on these findings, the incidence of neoadjuvant treatment related toxicity in elderly patients with LARC is indeed higher than that in younger patients, but it is quite acceptable. On the other hand, all patients in our study completed the radiotherapy procedure and only five patients experienced prolonged radiotherapy. This also indicated that neoadjuvant treatment is well tolerated in elderly patients with LARC. Surgery morbidity and mortality are another concerns for clinicians when performing surgery in elderly patients [16]. It was reported that the mortality rate after TME surgery was at least $2-5 \%$, and even higher in older patients [16]. With the improvement of surgical and anesthesiological techniques, postoperative nutritional support and physical activity interventions, postoperative morbidity and mortality in most elderly patients are no longer different from their younger counterparts [16]. In a prospective cohort study, the rates of postoperative complications for elderly patients and younger counterparts were $38.5 \%$ and $34.7 \%$ [10]. As for the present study, most TME surgeries were performed with laparoscopic technique $(85.4 \%)$, the postoperative morbidity rate was only $12.5 \%$.

The rate of $\mathrm{pCR}$ is an important factor for assessing the effect of neoadjuvant chemoradiotherapy and it is also related with improved survival outcomes [15]. The pCR rate is significantly influenced by the escalated dose to primary tumor. When receiving $\geq 60$ Gy irradiation, the pooled pCR rate was $20.4 \%$ in a mata-analysis [23]. A prospective study from China recruited 63 patients with LARC, patients received IMRT with pelvic to $41.8 \mathrm{~Gy}$ in 22 fractions and primary tumor to 50.6Gy simultaneously. The pCR rate was as high as $31.0 \%$ [24]. Zhu, et al also investigated the effect of concomitant boost irradiation on PCR rate in a phase II study [25]. The prescribed doses to the pelvic area and primary tumor were 50Gy in 25 fractions and 55Gy in 25 fractions, respectively. A total of 18 in 78 patients $(23.7 \%)$ obtained pCR after surgery. However, when patients 
were treated without primary tumor dose escalation, the reported pCR rates only varied from $11.4 \%$ to 19.2 [2, 26-28]. Regarding elderly patients with LARC, Jacobs L, et al retrospectively reviewed 42 patients aged $\geq 70$ years receiving neoadjuvant chemoradiotherapy, the irradiation dose to pelvic region was 50 Gy in 25 fractions without primary tumor boost, pCR was merely observed in 3 patients $(7.5 \%)$. In the present study, $91.8 \%$ of eligible patients received IMRT with boost irradiation to primary tumor of 55Gy in 25 fractions. The pCR rate was $21.4 \%$, which was consistent with the previous meta-analysis [23]. Another propensity-score matching analysis [21] reported that the $\mathrm{pCR}$ rate wasn't significantly different between elderly and younger patients (14.8\% vs $17.1 \%, \mathrm{P}=0.433$ ). Obviously, elderly patients can also get benefit from dose escalation radiotherapy as their younger counterparts do.

There is another key point that should not be ignored. With increasing age, more patients would die from other comorbidities [7], such as cardiovascular, nervous system and pulmonary diseases. Eight patients died of comorbidities instead of rectal cancer, accounting for $25.8 \%$ of all deceased in our study. Considering this, DSS, DFS and LC are more suitable for evaluating the efficacy of treatment in elderly patients with LARC. Our study reported that the 3-year and estimated 5-year DSS, DFS and LC for patients receiving multimodal therapy were $75.8 \%$ and $60.4 \%, 68.2 \%$ and $56.1 \%, 83.9 \%$ and $78.3 \%$, respectively. These results were quite comparable to the previous studies regarding elderly patients [12, 20-22, 29]. Notably, the oncologic outcomes of our study were not inferior than younger patients in other studies [2, 15, 21]. In the study of FFCD 9203 [2], the reported 5-year progression free survival (PFS) and local recurrence rate (LRR) were $59.4 \%$ and $8.1 \%$. Recently, Sung SY, et al [21] conducted a propensityscore matching study to compare oncologic outcome and morbidity between elderly and younger patients after neoadjuvant chemoradiotherapy and TME surgery. Though the rates of acute hematologic toxicity and late complications were higher in elderly patients, no significant difference was observed regarding 5-year recurrence free survival (RFS) between elderly and younger patients $(65.5 \%$ vs $67.7 \%, \mathrm{P}=0.483$ ). Thus, age may not be a determining factor when clinicians develop treatment strategies for elderly patients with LARC.

Watch-and-watch policy is an alternative for patients with $\mathrm{CCR}$ after neoadjuvant treatment, especially for elderly or frail patients [30-32]. With this approach, patients can avoid the inconvenience of colostomy and other surgery complications without the expense of survival, which may further led to a good quality of life [33]. A meta-analysis [31] enrolled 23 studies including 867 patients with respect to watch-and-wait strategy and surgery for patients with LARC. The pooled 2-year local regrowth was $15.7 \%$, and most patients (95.4\%) received salvage therapies. No significant difference was observed regarding non-regrowth recurrence, CSS, DFS and OS between patients with $\mathrm{CCR}$ after neoadjuvant treatment managed with watch-and-wait approach and surgery. Eleven patients obtained CCR after chemoradiotherapy in our study. Watch-and-wait approach was performed in seven patients. By the end of follow-up, only one patient suffered regrowth recurrence. The number of patients with CCR in present study was too small to draw meaningful conclusions, but to some extent, it showed promise to implement this approach in elderly patients with LARC.

Elderly patients were defined as patients older than 70 years in most of the previous studies $[9,12,18$, $21,22]$. However, rectal cancer mainly affects elderly people with a peak incidence of over 80 years [6]. When people reach their 80th year, there is still life expectancy of nearly 10 years for both men and women [20]. From this prospective, our study including patients 75 years or older might be more representable for geratic patients in rectal cancer. Limitations still exist in our study. The major limitation must be the retrospective nature, some patients' medical records were incomplete which might compromise our conclusions. Another shortage is the relatively small number of our study group. Though we included patients treated ten years ago (2008), few elderly patients with LARC underwent neoadjuvant treatment before 2012 at our institute.

\section{Conclusion}

In patients aged 75 years or older with LARC, neoadjuvant chemoradiotherapy followed by surgery was well tolerated with promising survival outcomes, which should be strongly suggested if medically suitable.

\section{Acknowledgements}

This study was supported by the National Key Research and Development Plan, the Ministry of Science and Technology of the People's Republic of China (grant number 2016YFC0105207).

\section{Competing Interests}

The authors have declared that no competing interest exists.

\section{References}

1. Bosset JF, Calais G, Mineur L, Maingon P, Radosevic-Jelic L, Daban A, et al. Enhanced tumorocidal effect of chemotherapy with preoperative radiotherapy 
for rectal cancer: preliminary results--EORTC 22921. J Clin Oncol. 2005; 23: 5620-7.

2. Gerard JP, Conroy T, Bonnetain F, Bouche O, Chapet O, Closon-Dejardin MT, et al. Preoperative radiotherapy with or without concurrent fluorouracil and leucovorin in T3-4 rectal cancers: results of FFCD 9203. J Clin Oncol. 2006; 24: 4620-5.

3. Sauer R, Liersch T, Merkel S, Fietkau R, Hohenberger W, Hess C, et al. Preoperative versus postoperative chemoradiotherapy for locally advanced rectal cancer: results of the German CAO/ARO/AIO-94 randomized phase III trial after a median follow-up of 11 years. J Clin Oncol. 2012; 30: 1926-33.

4. Roh MS, Colangelo LH, O'Connell MJ, Yothers G, Deutsch M, Allegra CJ, et al. Preoperative multimodality therapy improves disease-free survival in patients with carcinoma of the rectum: NSABP R-03. J Clin Oncol. 2009; 27: 5124-30.

5. Ngan SY, Burmeister B, Fisher RJ, Solomon M, Goldstein D, Joseph D, et al. Randomized trial of short-course radiotherapy versus long-course chemoradiation comparing rates of local recurrence in patients with $\mathrm{T} 3$ rectal cancer: Trans-Tasman Radiation Oncology Group trial 01.04. J Clin Oncol. 2012; 30: 3827-33

6. Matanoski G, Tao X, Almon L, Adade AA, Davies-Cole JO. Demographics and tumor characteristics of colorectal cancers in the United States, 1998-2001. Cancer. 2006; 107: 1112-20

7. Bhangu A, Kiran RP, Audisio R, Tekkis P. Survival outcome of operated and non-operated elderly patients with rectal cancer: A Surveillance, Epidemiology, and End Results analysis. Eur I Surg Oncol. 2014; 40: 1510-6.

8. Manceau G, Karoui M, Werner A, Mortensen NJ, Hannoun L. Comparative outcomes of rectal cancer surgery between elderly and non-elderly patients: a systematic review. Lancet Oncol. 2012; 13: e525-36.

9. Cai X, Wu H, Peng J, Zhu J, Cai S, Cai G, et al. Tolerability and outcomes of radiotherapy or chemoradiotherapy for rectal cancer in elderly patients aged 70 years and older. Radiat Oncol. 2013; 8: 86.

10. Couwenberg AM, de Beer FSA, Intven MPW, Burbach JPM, Smits AB, Consten ECJ, et al. The impact of postoperative complications on health-related quality of life in older patients with rectal cancer; a prospective cohort study. Journal of geriatric oncology. 2018; 9: 102-9.

11. Portale G, Valdegamberi A, Cavallin F, Frigo F, Fiscon V. Effect of Age and Comorbidities on Short- and Long-Term Results in Patients Undergoing Laparoscopic Curative Resection for Rectal Cancer. Journal of laparoendoscopic \& advanced surgical techniques Part A. 2019; 29: 353-9.

12. Francois E, Azria D, Gourgou-Bourgade S, Jarlier M, Martel-Laffay I, Hennequin C, et al. Results in the elderly with locally advanced rectal cancer from the ACCOR12/PRODIGE 2 phase III trial: tolerance and efficacy. Radiother Oncol. 2014; 110: 144-9.

13. Sun G, Xue J, Zhang Y, Gao X, Guo F. Short- and long-term outcomes of laparoscopic surgery in elderly patients with rectal cancer. Journal of BUON: official journal of the Balkan Union of Oncology. 2018; 23: 55-61.

14. Bujko K, Nasierowska-Guttmejer A, Wyrwicz L, Malinowska M, Krynski J, Kosakowska E, et al. Neoadjuvant treatment for unresectable rectal cancer: an interim analysis of a multicentre randomized study. Radiother Oncol. 2013; 107: 171-7.

15. Zhao J, Liu X, Wang W, Hu K, Zhang F, Hou X, et al. $<\mathrm{p}>$ Concomitant dose escalation with image\&ndash;guided Tomotherapy in locally advanced mid\&ndash;low rectal cancer: a single-center study $</ p>$. Cancer management and research. 2019; Volume 11: 1579-86.

16. Bujko K, Glynne-Jones R, Papamichael D, Rutten HJT. Optimal management of localized rectal cancer in older patients. Journal of geriatric oncology. 2018; 9: 696-704.

17. Rutten HJT, den Dulk M, Lemmens VEPP, van de Velde CJH, Marijnen CAM. Controversies of total mesorectal excision for rectal cancer in elderly patients. The Lancet Oncology. 2008; 9: 494-501.

18. Jacobs L, van der Vlies E, Ten Bokkel Huinink D, Bloemendal H, Intven M, Smits $\mathrm{AB}$, et al. Tolerability, Safety, and Outcomes of Neoadjuvant Chemoradiotherapy With Capecitabine for Patients Aged $>/=70$ Years With Locally Advanced Rectal Cancer. Clinical colorectal cancer. 2018; 17: 179-86.

19. Margalit DN, Mamon HJ, Ancukiewicz M, Kobayashi W, Ryan DP, Blaszkowsky LS, et al. Tolerability of combined modality therapy for rectal cancer in elderly patients aged 75 years and older. Int J Radiat Oncol Biol Phys. 2011; 81: e735-41.

20. Tougeron D, Roullet B, Paillot B, Hamidou H, Tourani JM, Bensadoun RJ, et al. Safety and outcome of chemoradiotherapy in elderly patients with rectal cancer: results from two French tertiary centres. Digestive and liver disease : official journal of the Italian Society of Gastroenterology and the Italian Association for the Study of the Liver. 2012; 44: 350-4.

21. Sung SY, Jang HS, Kim SH, Jeong JU, Jeong S, Song JH, et al. Oncologic Outcome and Morbidity in the Elderly Rectal Cancer Patients After Preoperative Chemoradiotherapy and Total Mesorectal Excision: A Multi-institutional and Case-matched Control Study. Annals of surgery. 2019; 269: 108-13.

22. De Felice F, Llange K, Rubini F, Bulzonetti N, Caiazzo R, Musio D, et al. Intensified Neoadjuvant Chemoradiotherapy for Locally Advanced Rectal Cancer in Elderly Patients: Toxicity, Disease Control, and Survival Outcomes. Clinical colorectal cancer. 2018; 17: e77-e81.

23. Burbach JP, den Harder AM, Intven $\mathrm{M}$, van Vulpen $\mathrm{M}$, Verkooijen $\mathrm{HM}$, Reerink O. Impact of radiotherapy boost on pathological complete response in patients with locally advanced rectal cancer: a systematic review and meta-analysis. Radiother Oncol. 2014; 113: 1-9.
24. Li JL, Ji JF, Cai Y, Li XF, Li YH, Wu H, et al. Preoperative concomitant boost intensity-modulated radiotherapy with oral capecitabine in locally advanced mid-low rectal cancer: a phase II trial. Radiother Oncol. 2012; 102: 4-9.

25. Zhu J, Liu F, Gu W, Lian P, Sheng W, Xu J, et al. Concomitant boost IMRT-based neoadjuvant chemoradiotherapy for clinical stage II/III rectal adenocarcinoma: results of a phase II study. Radiat Oncol. 2014; 9: 70.

26. Kim DY, Jung KH, Kim TH, Kim DW, Chang HJ, Jeong JY, et al. Comparison of 5-fluorouracil/leucovorin and capecitabine in preoperative chemoradiotherapy for locally advanced rectal cancer. Int J Radiat Oncol Biol Phys. 2007; 67: 378-84.

27. Jeong DH, Lee HB, Hur H, Min BS, Baik SH, Kim NK. Optimal timing of surgery after neoadjuvant chemoradiation therapy in locally advanced rectal cancer. Journal of the Korean Surgical Society. 2013; 84: 338-45.

28. Park IJ, You YN, Agarwal A, Skibber JM, Rodriguez-Bigas MA, Eng C, et al. Neoadjuvant treatment response as an early response indicator for patients with rectal cancer. J Clin Oncol. 2012; 30: 1770-6.

29. Wan JF, Zhu J, Li GC, Sun WJ, Zhang Z. Implications for determining the optimal treatment for locally advanced rectal cancer in elderly patients aged 75 years and older. Oncotarget. 2015; 6: 30377-83

30. Maas M, Beets-Tan RG, Lambregts DM, Lammering G, Nelemans PJ, Engelen $\mathrm{SM}$, et al. Wait-and-see policy for clinical complete responders after chemoradiation for rectal cancer. J Clin Oncol. 2011; 29: 4633-40.

31. Dossa F, Chesney TR, Acuna SA, Baxter NN. A watch-and-wait approach for locally advanced rectal cancer after a clinical complete response following neoadjuvant chemoradiation: a systematic review and meta-analysis. The lancet Gastroenterology \& hepatology. 2017; 2: 501-13.

32. Appelt AL, Pløen J, Harling H, Jensen FS, Jensen LH, Jørgensen JCR, et al. High-dose chemoradiotherapy and watchful waiting for distal rectal cancer: a prospective observational study. The Lancet Oncology. 2015; 16: 919-27.

33. Renehan AG, Malcomson L, Emsley R, Gollins S, Maw A, Myint AS, et al. Watch-and-wait approach versus surgical resection after chemoradiotherapy for patients with rectal cancer (the OnCoRe project): a propensity-score matched cohort analysis. The Lancet Oncology. 2016; 17: 174-83. 